Bond University

Research Repository

\title{
Analysis of articles directly related to randomized trials finds poor protocol availability and inconsistent linking of articles
}

Sender, David; Clark, Justin; Hoffmann, Tammy

Published in:

Journal of Clinical Epidemiology

DOI:

10.1016/j.jclinepi.2020.04.017

Licence:

CC BY-NC-ND

Link to output in Bond University research repository.

Recommended citation(APA):

Sender, D., Clark, J., \& Hoffmann, T. (2020). Analysis of articles directly related to randomized trials finds poor protocol availability and inconsistent linking of articles. Journal of Clinical Epidemiology, 124, 69-74.

https://doi.org/10.1016/j.jclinepi.2020.04.017

\section{General rights}

Copyright and moral rights for the publications made accessible in the public portal are retained by the authors and/or other copyright owners and it is a condition of accessing publications that users recognise and abide by the legal requirements associated with these rights.

For more information, or if you believe that this document breaches copyright, please contact the Bond University research repository coordinator 
Title:

Analysis of articles directly related to randomised trials finds poor protocol availability and inconsistent linking of articles

Author names and affiliations:

David Sender ${ }^{a}$, Justin Clark ${ }^{a}$, Tammy Hoffmann ${ }^{a}$

a Institute for Evidence-Based Healthcare, Faculty of Health Sciences and Medicine, Bond University, Gold Coast, Australia, 4226

Corresponding author:

Professor Tammy Hoffmann

Institute for Evidence-Based Healthcare

Faculty of Health Sciences and Medicine

Bond University

Robina, QLD, Australia, 4226

Phone: +61755955522

thoffman@bond.edu.au

Declaration of interests: none 


\section{ABSTRACT}

Objective: Interpreting a randomised trial requires access to more than the main results paper. We aimed to determine the: 1) proportion of trials referring to the protocol in the trial report and their accessibility; 2) proportion of protocols accessible from trial registry entry and by trial registration number search; and 3) types of additional publications associated with trial reports.

Study Design and Setting: A previously-gathered sample of randomised trials of nonpharmacological interventions published in 2009 was used. Trial reports and registry entries were searched for protocol mentions and obtained when possible. Related publications were identified using citation searching.

Results: Of 133 trials, 96 (72\%) mentioned the protocol within the report, 61 (64\%) contained details about protocol acquisition, with 48 (36\%) protocols obtainable. Of the 129 registered trials, 32 (25\%) had protocols obtainable from registry entry. Citation tracking identified 1030 related publications; secondary analyses and qualitative studies most common.

Conclusion: Trial protocols facilitate good trial conduct and interpretation. However, they are often not linked to the main report or registry and can be difficult to obtain. Many trials have related publications which are inconsistently linked. Trial registries and registration numbers could facilitate the threading of articles related to a trial, but currently do not.

\section{Keywords:}

randomized controlled trial; trial registration; protocol; registries; records

\section{Running Title:}

Randomised trials: protocol availability and threaded articles

Word Count: 2654 


\section{What's New}

- Analysis of the linking of protocols to main trial reports and trial registry entries

- Only one in three randomised trials had an obtainable protocol

- Protocols obtainable from trial registry entry for only $25 \%$ of trials

- Many randomised trials had numerous related publications, but they are inconsistently linked to the trial report

- Trial registries and registration numbers could facilitate threading, but do not 


\section{Background}

Randomised controlled trials are the gold standard for estimating treatment effects as they are less susceptible to bias and confounding than other primary study designs [1]. Trials require substantial financial and non-financial resources and time to conduct. While publication of the results of a trial is typically in the form of a main results article in a journal, many trials generate multiple related publications. The concept of 'linked or threaded publications' was proposed by Chalmers and Altman [2] in 1999. It considers that the published main trial report is one element in a series of 'threaded' publications that link together the trial protocol, main results paper (the primary trial paper), and other related papers, such as commentaries, secondary analyses, economic/cost analyses, follow-up studies, and systematic reviews [3].

Awareness of these other linked publications by readers of a trial is low and there is difficulty accessing the additional publications that surround an individual trial [4]. All the related publications for a trial are rarely available from a single resource and locating them usually requires labour-intensive searching in multiple sources such as bibliographic databases, trial registries, author websites, and regulatory review reports [5]. When publications and complete trial details and data are not publicly available, trial authors need to be contacted - although this often does not produce a response or the required information [6].

Good trial protocols are important for facilitating appropriate conduct and reporting of randomised trials, and their availability contributes to minimising research waste $[7,8]$. [8].Protocols are not publicly available for many trials, but an estimate of the extent of this 
has not been previously quantified. Arguably, the most potentially powerful strategy for linking trial protocols, trial reports, and other related publications is trial registration. However, this strategy appears to be grossly underutilised. Despite mandates by organisations, including the International Committee of Medical Journal Editors (ICMJE) requiring timely trial registration with an acceptable registry, [9] about half of trials are not registered [10]. Even for registered trials, it is uncommon for protocols or other related documents, to be provided as part of the registration process, with few trial registry systems requiring, checking, or even enabling upload of trial materials [11].

The aims of this study were to determine, in a sample of randomised trials of nonpharmacological interventions: 1) the proportion of trials that referred to the trial protocol in the main trial report and accessibility of the protocols; 2 ) the proportion of trial protocols that could be obtained by searching using a trial's registration number; and 3) the types of additional publications associated with main trial reports that could be identified through searching.

\section{Methods}

\section{$\underline{\text { Search strategy and selection of trial reports }}$}

A previously collected sample [6] of 133 screened and eligible randomised controlled trials of non-pharmacological interventions published in 2009 in one of the six leading general medical journals (based on ISI Web of Knowledge Impact Factor for 2010) - New England Journal of Medicine, the Journal of the American Medical Association, Lancet, Annals of Internal Medicine, PLOS Medicine, and the British Medical Journal, was used. This sample had been identified using a search of PubMed in April 2011, using the restrictions of year 
(2009), publication type ('randomised controlled trial'), and journal title (the six chosen journals). As part of the previous study [6], two researchers screened the 358 titles and abstracts retrieved, identified reports that might meet the inclusion criteria, and retrieved the full text $(n=133)$.

\section{Procedure for data collection}

For each trial, data were collected and recorded by one researcher (DS), in a data extraction form developed in Microsoft Excel.

Trial protocols: To identify whether the trial report mentioned the trial protocol, text searching of the PDF, via search functionality, of each trial report was conducted. Text words searched for were: 'protocol', 'earlier', 'previous', 'elsewhere'. If the protocol was mentioned, details were noted about where it was mentioned within the full-text, and details about how to access the protocol (such as a journal reference, URL, trial registry number, supplementary material, trial or author's website, or by request from the author). Efforts were made to obtain the full text of the protocol of each trial, using the details provided in the trial report, and whether it could be obtained was recorded. The trial registry number and name of registry were obtained for all registered trials, the relevant trial registry was accessed, and the unique trial registry page for each trial was manually searched for a protocol (such as link to, reference to, or file upload).

Additional publications related to the main trial: Two strategies were used by a medical librarian to identify additional publications, with these searches conducted between October 2018 and July 2019. Firstly, each trial's registration number was searched in PubMed, all results found were imported into EndNote and labelled with the study identifier 
of the original trial (first author's surname and publication year), as well as the search method used to identify it (trial registry number search). Secondly, the title of each study was searched for and located in Scopus, then a backward and forward citation search for each trial was performed.

All search results were grouped in EndNote by original trial report and one group was examined at a time to determine if the publication related to the original trial. Duplicates among each group were deleted and all search results were labelled with the original trial report and the search method used to find it (trial registry number, forward or backward citation). Any article from the forward and backward citation analysis that did not contain at least one of the authors from the main trial report was excluded. Where there were 10 or more authors, this was restricted to requiring only the first or senior (last) author to be listed, or in the case of large authorship group (>100 authors) trials, papers were examined if they had either one of the first ten authors or any of the last three authors. The abstract and full text of articles identified by this search process were examined by a researcher (DS), with discussion with an additional assessor (TH or JC) if needed. Articles assessed as being related to the main trial report were categorised into type of study.

\section{Results}

\section{$\underline{\text { Reference to and accessibility of trial protocols }}$}

Figure 1 shows the proportion of main trial reports that mentioned the trial protocol and whether the protocols could ultimately be obtained. Of the 133 trials, 96 (72.2\%) mentioned the trial protocol within the main trial report. Of these 96,61 (63.5\%) contained details about how to obtain the protocol (such as a website, email to corresponding author, 
reference, trial registry, or appendix/supplementary material). Forty-eight (78.7\%) of these protocols were able to be obtained. Thirteen protocols were unable to be obtained due to non-functioning web links, insufficient location details provided, or non-response from authors who had advised that protocols were available upon email request. For the 133 trials, 48 (36.1\%) protocols were accessible.

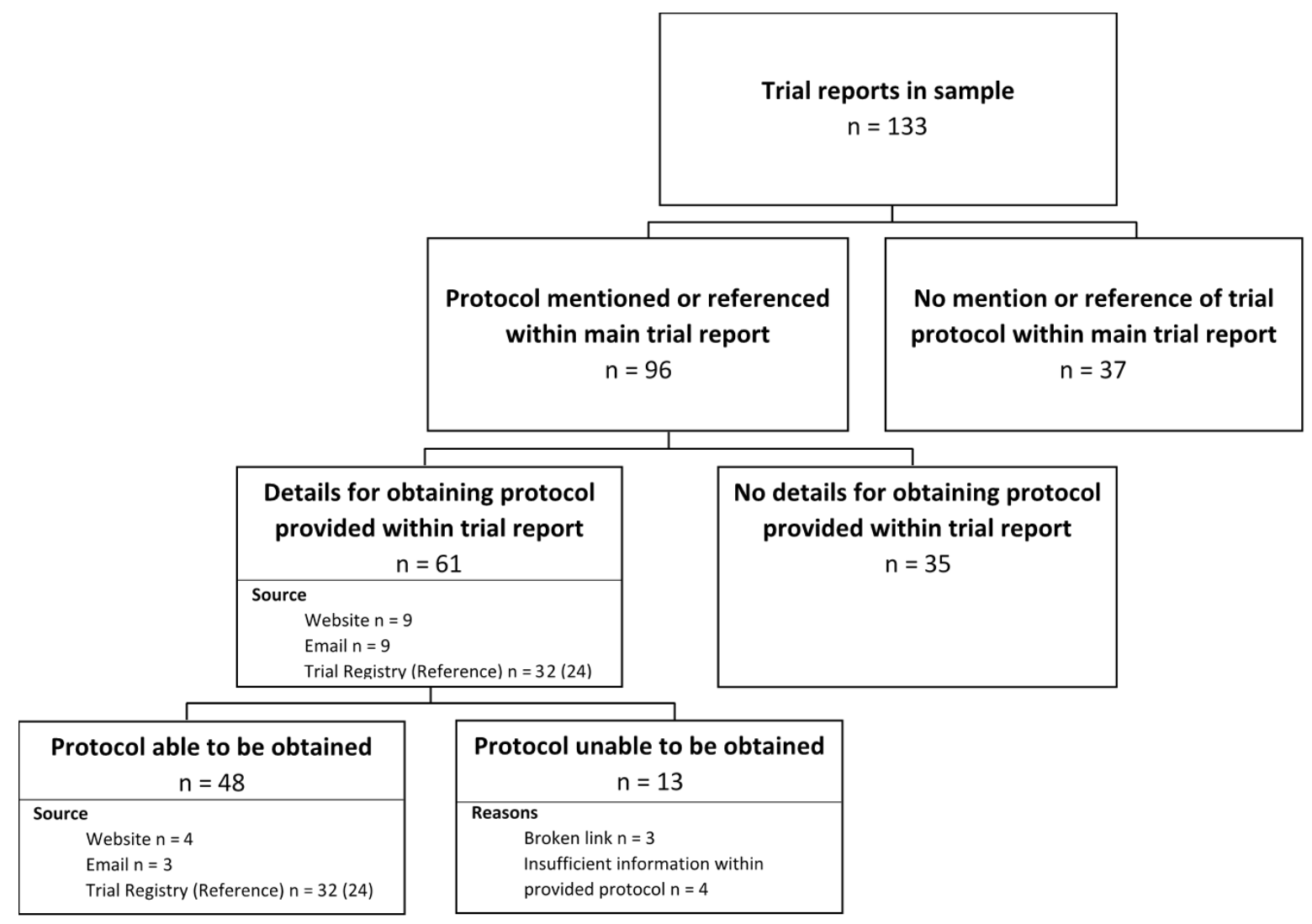

Figure 1. Flowchart showing the proportion of trials that mentioned a protocol and accessibility of the protocol

\section{$\underline{\text { Trial registry search results }}$}

Of the 133 trials, 129 (97.0\%) were registered, and 1 trial reported that it was exempt from registration. The majority $(77,59.7 \%)$ of these were registered in the Clinicaltrials.gov registry, 49 (38.0\%) with the ISRCTN Registry, 2 with the Australian and New Zealand Clinical 
Trials Registry, and 1 trial in the Chinese University of Hong Kong Clinical Trial Registry. Of the 129 registered trials, $32(24.8 \%)$ had protocols accessible through their associated trial registry. Thirty of these were links to a citation in PubMed of the trial's published protocol (published prior to publication of the main trial report). The other two protocols were obtained as a link to the protocol on the trial's website was provided. None of these protocols were ones that were not also found by searching the main trial report. There was little difference between the main two registries in terms of protocol availability (ISRCTN $n=$ 14, Clinicaltrials.gov $n=18)$.

\section{Additional publications identified by forward and backward citation analysis}

The backwards and forwards citation search yielded a total of 4928 publications, of which 1030 remained after removal of duplicates and deletion of trials not directly related to one of the 133 trials in our sample. Table 1 shows the number of additional studies located by the searching and the types of studies identified.

Of the 1030 total additional studies located, across the 133 trial reports, 143 (13.8\%) were published before the publication date of the main trial report and comprised of four study types: pilot studies or preliminary findings, protocols, qualitative studies, and economic analyses. Of the 887 additional publications detected in the forward citation search (published after the publication date of the main trial report), the three most common study types were a further analysis of the trial data, follow-up results, and qualitative studies. 


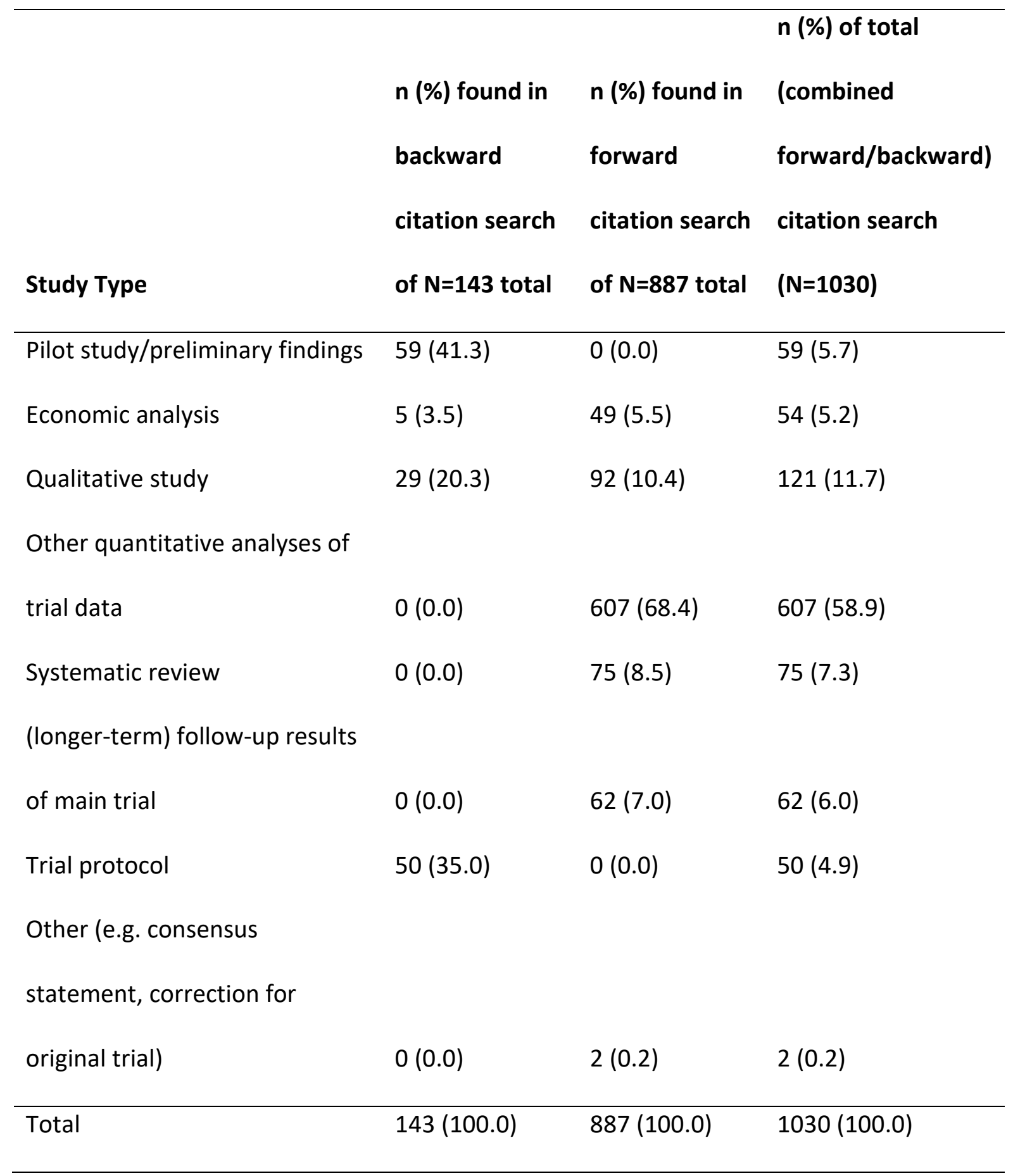

Table 1. Study types identified through backward and forward citation searching of the randomised trials 


\section{Discussion}

This study aimed to assess the accessibility of trial protocols from the trial main report and trial registry, along with exploration of the types of additional publications that are associated with reports of trials of non-pharmacological interventions. A protocol could be obtained for about one-third of the trials in our sample, with more protocols obtainable using details within the published trial's main report than in its registry entry. Most trials in our sample had a number of additional related publications, with subsequent quantitative analyses and qualitative studies being the most common types.

Although the majority of trials (72\%) in our sample mentioned their protocol within the main trial report, protocols were only able to be obtained for just over one-third of the trials. Many trials mentioned the existence of a trial protocol, but insufficient detail about how and where to obtain the protocol was missing for about one-third of them. The most frequent and successful method for obtaining a trial's protocol were from trials that had published their protocol and referenced it. Other methods were obtaining the protocol as part of the main trial report's supplementary material, email to the author, and via a provided weblink. The latter two often did not work, with broken weblinks or no response from authors. As our sample of trials was from 2009 and this analysis occurred in 2019, over a 10-year period some weblinks and emails have changed or ceased to exist, and as such data shared via these methods are highly susceptible to becoming unavailable.

Searching trial registry entries did not produce more protocols, with only onequarter of the trials having a protocol accessible (typically via a link to the published protocol) from the registry. Backwards citation searching found 50 study protocols, which is a similar proportion to the number of protocols found by searching the main trial reports. Ideally, all trials should make their protocol available (through either publication in a journal 
and in the trial's registry entry) and reference the protocol and its location in the main trial report.

The majority of publications found in the forwards citation search were other quantitative analyses of trial data. This presents an issue for unthreaded articles, as sometimes the results presented in other quantitative analyses of trial data can supplement earlier findings. For example, follow-up studies (with longer-term data) comprised $7 \%$ of the citations found by forward searching in our sample. If unthreaded to the original trial, data can easily be missed in syntheses. This can then confound subsequent syntheses such as systematic reviews. Our finding is similar to that of an analysis of a sample of 191 randomised trials [12] which found that about half had a large number of secondary publications and that many of these had additional, and statistically significant, results. Cochrane groups have been tackling this issue with the use of study-based registers [13] in which a record that links related records to a single meta-record is created. This metarecord then contains data tables with all the tabulated data from the multiple reports into a primary dataset [14]. Others, including publishers, have been attempting to tackle this issue by increasing the publication of study protocols, and working closely with both trial registries and databases to further develop existing infrastructure to capture the metadata about an article [3].

The finding in our study of being able to obtain about one-third of protocols is similar to the finding of a meta-epidemiologic study [15] which compared the analysis plan in trial reports to the plan stated in the protocol and found that that $40 \%$ of protocols were accessible from a sample of 199 articles. Author response rate to email contact was approximately a third of those contacted, which is similar to other studies $[12,15]$, however in these studies, corresponding authors of all articles were contacted. The availability of 
protocols does not seem to be improving over time, with an analysis of trials submitted to The Lancet in 2016 finding that only 95 (36\%) of the 216 submissions had a protocol that was publicly available [16].

Our study specifically looked at the accessibility of a trial protocol - this is important as there is evidence to suggest that selective reporting of outcomes occurs in randomised trials $[17,18]$ and this can be detected by comparing protocols to the main published trial report. These studies suggested the use of the trial registry as a means for facilitating protocol accessibility. However, our study found that only one-quarter of the trials had accessible protocols from the registry. The only obligation from ICMJE requirements is reporting of the trial registry ID in the article abstract when reporting trial outcomes [19]. This identifies the key issue with regards to a reference-based registry, where each citation acts individually, and unless a record contains a link somehow, such as via the trial registry number, additional citations do not appear as threaded within the registry [4].

One of the study limitations is that our sample only contained trials of nonpharmacological interventions and only trials from the top six leading general medical journals. These journals typically adhere more strictly to ICMJE requirements, such as requiring trial registration, than lower impact journals and hence our results may be an overestimate of the proportion of trials that have accessible protocols. Furthermore, trials in these journals are more likely to have generated a high number of forward citations of the original trial report [12]. This effect may overestimate the number of additional relevant publications that exist for trials. Exploration of the accessibility of protocols and additional relevant publications in a sample that includes trials of pharmacological interventions and those published in journals beyond the major general medical journals is needed. A further limitation is that our sample of trials was from 2009, and in subsequent years there has 
been increased awareness about the importance of study protocols as well as publication of the Standard Protocol Items: Recommendations for Interventional Trials (SPIRIT) reporting guideline [7]. However, as we were exploring trial-related papers that are published after the main trial report, there was also a need to ensure there was a reasonable number of years between the publication of the main trial reports and our search. Our study did not analyse the protocol to assess for discrepancies between the protocol content and the published data, and only one author searched trial registries and screened the search results for relevant additional publications.

\section{Conclusion}

Threaded publications for randomised trials, in which the study protocol is the often first element in a series of a threaded articles, followed by the main trial report and secondary publications, is not a new idea [2]. However, little progress towards achieving it appears to have occurred since the idea was first proposed, and the substantial fragmentation of publications related to a trial is a contributor to waste in research. Trial protocols are often not linked to, or mentioned in, the main trial report or its registry entry and can be difficult or impossible to obtain. Many trials have a number of related publications, but these are not consistently linked to the trial. Trial registries and registration numbers provide an opportunity to facilitate the threading process of articles linked to a randomised trial but are currently underutilised. 


\section{Declarations:}

Availability of data and materials

- The datasets used and/or analysed during the current study are available from the corresponding author on reasonable request

Competing interests

- The authors declare that they have no competing interests.

Funding

- This research did not receive any specific grant from funding agencies in the public, commercial, or not-for-profit sectors.

Author's contributions

- DS performed the data extraction and analysis and led the writing of the first draft of the manuscript. JC conducted the searches and assisted with data extraction. TH was responsible for conception of the project and assisted with data analysis and interpretation. All authors read and approved the final manuscript.

Acknowledgements

- $\quad$ TCH is supported by a National Health and Medical Research Council of Australia (NHMRC) Senior Research Fellowship. The funders had no role in design and conduct of the study; collection, management, analysis, and interpretation of the data; and preparation, review, or approval of the manuscript. The authors thank Professor Paul Glasziou for his comments on a draft manuscript. 


\section{References}

1. Goldacre B, Gray J. OpenTrials: towards a collaborative open database of all available information on all clinical trials. Trials. 2016;17:164.

2. Chalmers I, Altman DG. How can medical journals help prevent poor medical research? Some opportunities presented by electronic publishing. Lancet. 1999;353(9151):490-3.

3. Altman DG, Furberg CD, Grimshaw JM, Shanahan DR. Linked publications from a single trial: a thread of evidence. Trials. 2014;15:369.

4. Bashir R, Bourgeois FT, Dunn AG. A systematic review of the processes used to link clinical trial registrations to their published results. Systematic reviews. 2017;6(1):123.

5. Mayo-Wilson E, Li T, Fusco N, Dickersin K. Practical guidance for using multiple data sources in systematic reviews and meta-analyses (with examples from the MUDS study). Research synthesis methods. 2018;9(1):2-12.

6. Hoffmann TC, Erueti C, Glasziou PP. Poor description of non-pharmacological interventions: analysis of consecutive sample of randomised trials. BMJ. 2013;347.

7. Chan AW, Tetzlaff JM, Gotzsche PC, Altman DG, Mann H, Berlin JA, et al. SPIRIT 2013 explanation and elaboration: guidance for protocols of clinical trials. BMJ. 2013;346:e7586.

8. Glasziou P, Altman DG, Bossuyt P, Boutron I, Clarke M, Julious S, et al. Reducing waste from incomplete or unusable reports of biomedical research. Lancet.

2014;383(9913):267-76.

9. ICMJE Recommendations for the Conduct, Reporting, Editing and Publication of Scholarly Work in Medical Journals [Available from: http://www.icmje.org/abouticmje/faqs/clinical-trials-registration/.

10. Macleod MR, Michie S, Roberts I, DirnagI U, Chalmers I, loannidis JP, et al. Biomedical research: increasing value, reducing waste. Lancet (London, England). 2014;383(9912):101-4.

11. Hoffmann T, Glasziou P, Beller E, Goldacre B, Chalmers I. Focus on sharing individual patient data distracts from other ways of improving trial transparency. BMJ.

2017;357:j2782.

12. Ebrahim S, Montoya L, Kamal El Din M, Sohani ZN, Agarwal A, Bance S, et al. Randomized trials are frequently fragmented in multiple secondary publications. Journal of Clinical Epidemiology. 2016;79:130-9.

13. Shokraneh F, Adams CE. Study-based registers of randomized controlled trials: Starting a systematic review with data extraction or meta-analysis. Biolmpacts : Bl. 2017;7(4):209-17.

14. Shokraneh F, Adams CE. Increasing value and reducing waste in data extraction for systematic reviews: tracking data in data extraction forms. Systematic reviews. 2017;6(1):153.

15. Saquib N, Saquib J, loannidis JPA. Practices and impact of primary outcome adjustment in randomized controlled trials: meta-epidemiologic study. BMJ. 2013;347:f4313.

16. Lucey M, Clark J, Glasziou P. Public availability of trial protocols. Lancet. 2017;390:e54-5.

17. Chan A-W, Pello A, Kitchen J, Axentiev A, Virtanen JI, Liu A, et al. Association of trial registration with reporting of primary outcomes in protocols and publications JAMA.

2017;318(17):1709-11. 
18. Chan AW, Hrobjartsson A, Haahr MT, Gotzsche PC, Altman DG. Empirical evidence for selective reporting of outcomes in randomized trials: comparison of protocols to published articles. JAMA. 2004;291(20):2457-65.

19. Huser V, Cimino JJ. Linking ClinicalTrials.gov and PubMed to track results of interventional human clinical trials. PloS one. 2013;8(7):e68409. 